Author generated pre-print of article accepted in Advanced Materials

DOI: $10.1002 / \mathrm{adma} .201504264$

\title{
Versatile soft grippers with intrinsic electroadhesion based on
}

\section{multifunctional polymer actuators}

Jun Shintake, Samuel Rosset, Bryan Schubert, Dario Floreano and Herbert Shea*

EPFL, Switzerland

For official version, please see http://dx.doi.org/10.1002/adma.201504264

Link to movie from supporting information https://goo.gl/IYXnX0
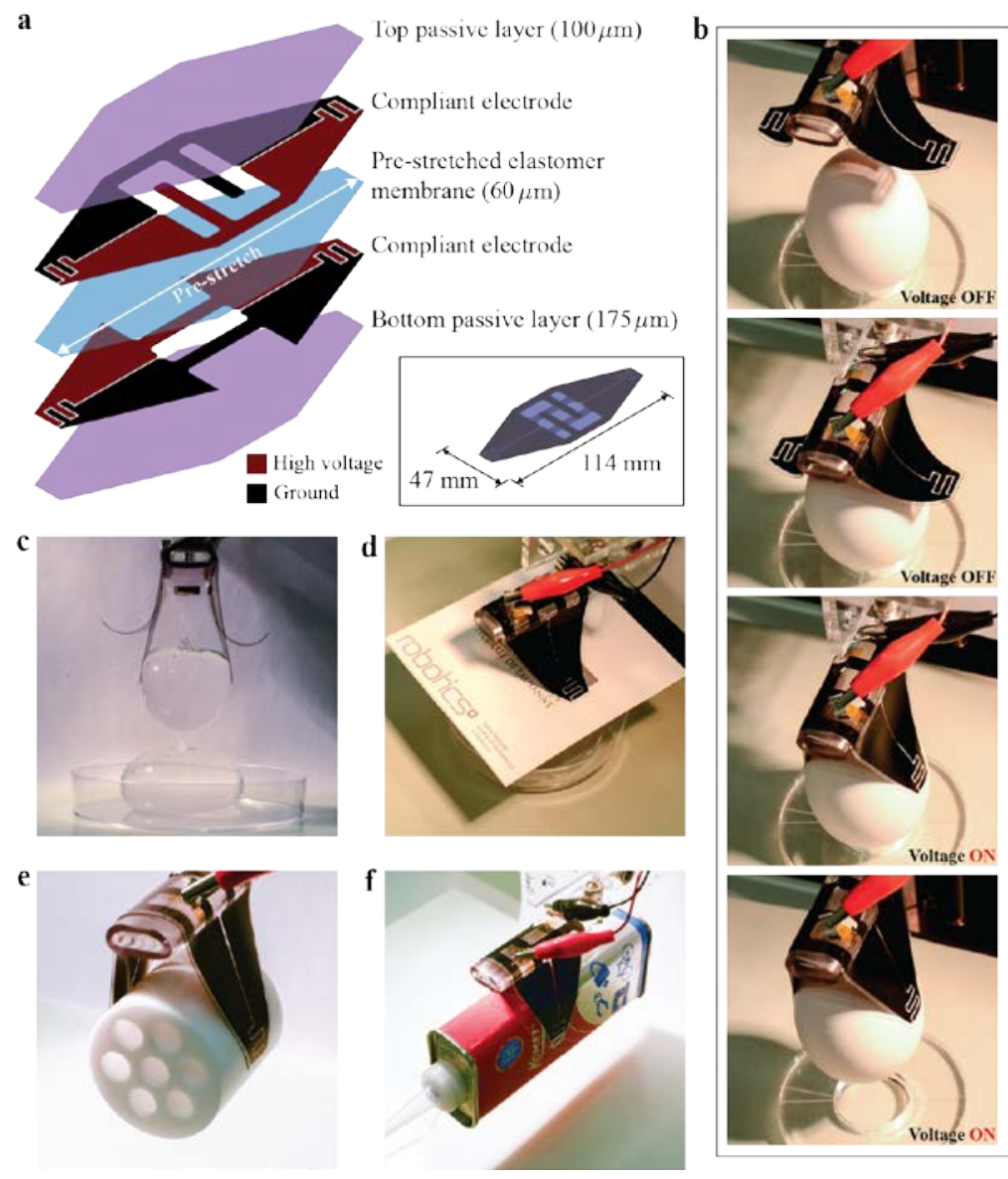


\section{Versatile soft grippers with intrinsic electroadhesion based on multifunctional polymer actuators}

Jun Shintake, Samuel Rosset, Bryan Schubert, Dario Floreano and Herbert Shea*

J. Shintake, Dr. S. Rosset, Prof. H. Shea

Institute of Microengineering Neuchâtel campus, School of Engineering, École Polytechnique Fédérale de Lausanne, Rue de la Maladière 71b, 2000 Neuchâtel, Switzerland Email: herbert.shea@epfl.ch

J. Shintake, Dr. B. Schubert, Prof. D. Floreano

Institute of Microengineering Lausanne campus, School of Engineering, Station 17, École Polytechnique Fédérale de Lausanne, 1015 Lausanne, Switzerland

Keywords: soft grippers, dielectric elastomer actuators, soft robotics, electroadhesion, multifunctional actuators

A child easily picks up with his hand objects of an enormous variety of shapes, even if they are fragile or highly deformable. In order to mimic this dexterity, fingered robotic grippers often use many actuators in combination with feedback control based on visual, tactile, force and angular position sensing, followed by trajectory planning ${ }^{[1-3]}$. If the target object is fragile, such as a chicken egg for example, the human hand adapts the grasping force, exploiting the compliance of tissue in the fingers and sensing the shear movement to safely grip the object ${ }^{[4]}$. Moreover, in the case of deformable objects such as a water balloon, human hands can sense the stiffness and follow the deformation while continuously regulating force and finger position. Replicating these grasping properties with traditional robotic grippers typically require complex sensing, motor accuracy, modelling, and learning ${ }^{[5-9]}$. Endowing robotic 
grippers made with rigid components with the versatility to grasp objects with many different shapes and differing material properties can be computationally challenging, costly and slow, and require external sensors. For this reason, robotic grippers typically have a specialized design for a specific object type ${ }^{[10]}$. Soft robotics ${ }^{[11,12]}$, especially when combined with artificial skins for distributed pressure and force sensing ${ }^{[11,13]}$, offer a path towards structures where the intrinsic compliance of elastomers allows for handling complex objects with simpler control thanks to passive adaptation. Early investigation on shape-adaptable grippers exploited a variety of technologies such as granule-filled bag from which air is evacuated ${ }^{[14]}$ (now known as granular jamming ${ }^{[15]}$ ), electrorheological (ER) fluids ${ }^{[16]}$, ER fluid with electroadhesion $^{[17]}$, pneumatic actuators ${ }^{[18]}$, and shape memory foams ${ }^{[18]}$. Yet even for current soft robots, many tasks that are easy for human hands, such as handling flat objects and deformable objects, remains a major challenge.

We demonstrate here a novel method to create soft grippers based on electrostatic actuation with intrinsic electroadhesion force, which enables them to manipulate deformable, fragile objects of any shape with a single control signal, and even flat objects like paper. We validate the method with a two-fingered gripper, shown in Figure 1, weighing $1.5 \mathrm{~g}$, that can pick up a wide range of objects, including: a fragile, highly-deformable water-filled thin membrane balloon (35.6 g), a raw chicken egg (60.9 g), a flat sheet of paper $(0.8 \mathrm{~g})$, a Teflon tube $(80.8$ $\mathrm{g})$, and a metallic can $(82.1 \mathrm{~g})$. These unprecedented capabilities are enabled by simultaneously maximizing electroadhesion and electrostatic actuation, while allowing selfsensing in a new design of dielectric elastomer actuators (DEAs) employing an interdigitated electrode geometry. The mechanical gripping force produced by electrostatic actuation is low $(1 \mathrm{mN})$, thereby allowing handling of very fragile objects, while the electroadhesion force can produce high holding force $\left(3.5 \mathrm{~N}\right.$ shear force for $\left.1 \mathrm{~cm}^{2}\right)$, thereby enabling the lifting of heavy objects. The electroadhesion force also enables the picking up of flat and deformable objects. 
The gripper can be controlled only by a single control voltage thanks to the simple, compliant composite structure, similar in overall concept to ${ }^{[15,19-24]}$. These features lead to a highlyintegrated, multifunctional conformal soft gripper with fast motion ( $\sim 100 \mathrm{~ms}$ to close fingers), high holding force and simplified structure and control, paving a way for sensitive, highly versatile grippers for soft robotics.

A key novelty, essential to reach human-like performance, is the simultaneous optimization of both electroadhesion and electrostatic actuation of a bending DEA by a novel electrode arrangement, allowing both in-membrane and fringing electric fields to be maximized, generating over 10 times higher electroadhesion force than would be obtained from a conventional DEA electrode configuration. The device is highly versatile, as bending motion and electroadhesion forces can be turned on one at a time or both simultaneously simply by applying suitable control voltages, yet the device retains a very simple architecture.

DEAs are an electrostatically-actuated type of electroactive polymer material ${ }^{[25,26]}$. They are compliant ( 1 MPa of elastic modulus), fast (response time less than $200 \mu \mathrm{s}^{[27,28]}$ ), and capable of self-sensing the actuator deformations ${ }^{[29]}$. The DEA structure consists of a thin elastomer membrane sandwiched between two highly compliant soft electrodes ${ }^{[30]}$. When a voltage is applied across the membrane thickness (electric fields typically 50 to $100 \mathrm{~V} / \mu \mathrm{m}$; for the gripper described here, $3.5 \mathrm{kV}$ is applied across a $60 \mu \mathrm{m}$-thick silicone membrane), opposite charges on the electrodes generate an electrostatic pressure (the Maxwell stress) leading to compression of the structure in the thickness direction and expansion in the planar directions, which can lead to bending. The Maxwell stress is proportional to the square of the electric field in the elastomer membrane in the direction normal to the surface ${ }^{[31]}$. For DEA actuation, one generally only considers this normal electric field in the elastomer. However the charges on the electrodes also create fringe electric fields at the edges of the electrodes. 
These fringe fields can generate electroadhesion in nearby objects, as the electric fields induce polarization of surface charges on the objects, resulting in attractive forces to the charges on the electrodes. In DEA applications, these forces are usually ignored and their use has not been reported to date.

Electroadhesion has been used in wafer handling ${ }^{[32]}$, wall climbing robots ${ }^{[33,34]}$, rigid and flexible grippers ${ }^{[35,36]}$. Early development also showed an end effector composed of a rigid flat substrate and an ER fluid encapsulated membrane ${ }^{[17]}$. Electroadhesion has many advantages over comparable adhesion technologies, such as vacuum or gecko-inspired adhesion. Vacuum handling technologies are widely used in industry, but are limited to objects with smooth, non-porous surfaces. Additionally, the need for vacuum pump and tubing adds significant bulk. Gecko-inspired adhesion uses van der Waals forces between passively deformed micro fibers and the target surface. However, in this technology, the adhesion mechanism relies upon external mechanical preloading in the normal or shear direction $^{[21,37-41]}$, which may cause unpredictable deformation and possible damage when the target object is deformable or fragile. Reversibility of the gecko adhesion is also challenging to achieve with lightweight objects, and the adhesion mechanism works poorly on low surface-energy materials, such as Teflon ${ }^{[42]}$. Electroadhesion can be used with smooth and rough surfaces, with dielectric objects and with metallic objects ${ }^{[32,35]}$ and is fully electrically controlled, obviating the need for additional actuators to pick up or release an object. A potential drawback is a small residual adhesion force after removing the voltage. However, these residual forces are reported to be extremely low for dielectric objects, and last for only a few seconds ${ }^{[43]}$. For conductive objects the small residual force can be greatly reduced by operating at low frequency AC voltage rather than $\mathrm{DC}^{[32]}$. 
The novelty of our method-a simple and effective method for simultaneously maximizing both electroadhesion and electrostatic actuation of a DEA actuator-lies in the segmentation of the compliant electrodes into a bilayer offset interdigitated four electrode geometry (Figure 2a), in contrast to conventional DEA designs that have only 2 uniform parallel electrodes. In order to maximize the electroadhesion force and the electrostatic actuation, the electrodes are wired such that adjacent electrode segments on the same planar surface are at opposite potentials, as are those electrodes that overlap each other across the membrane (Figure $2 \mathrm{~b}$ ). When a voltage bias is applied across the electrodes, fringe electric fields are generated at the segmentation boundaries providing homogeneous adhesion forces over the entire surface (Figure $2 \mathrm{~b}, \mathrm{c}$ ), contrary to conventional DEA designs that can generate adhesion forces only at the periphery. The same applied voltage provides electrostatic actuation in the region where electrodes directly overlap. The 4 electrode configuration enables the same device to generate: a) simultaneous electroadhesion and DEA actuation, or b) pure DEA actuation only (with no electroadhesion), or c) electroadhesion only (with no DEA actuation) simply by applying the appropriate voltages to the 4 electrodes (as shown in Supplementary Figure S1), thus allowing the same device to be reconfigured for different applications by applying control voltages. The passive silicone layers, sandwiching the DEA (Figure 2a), serve to insulate the electrodes to avoid electrical breakdown between adjacent electrodes or short-circuit via the external object. The bottom passive layer is in contact with the object being picked up. For these passive layers, a stiffer elastomer, Sylgard 184 ( 2.6 MPa of elastic modulus with curing temperature at $150 \mathrm{C}^{\mathrm{o}[4]}$ ) is used to minimize inherent tackiness, allowing smooth releasing of objects. The bending actuation of the gripper fingers is obtained by using a uniaxially prestretched DEA bonded to one or two passive layers (Figure $2 \mathrm{~d})^{[45]}$, similar to ${ }^{[46]}$. In this configuration, the structure has a curled shape at zero applied voltage where the internal stress of the pre-stretched DEA and the bending moment of the passive layers are at their equilibrium state. When a voltage is applied, the electrostatic pressure reduces the internal 
stress in the DEA and releases the bending moment in the passive layers, leading to voltagecontrollable bending actuation towards a flat shape. The initial bending angle, the actuation stroke, and the actuation force can be modulated by the mechanical parameters, such as, elastic modulus and thickness of both the DEA and the passive layers, and the DEA prestretch. The larger percentage change in capacitance of the device as it uncurls enables selfsensing of the bending angle (Supplementary Figure S2), as reported for other DEAs.

The electroadhesion force is proportional to the fringing fields, which are generated primarily at the electrode edges. For a given area, the total edge length for the interdigitated electrode is much longer than that of a uniform (i.e., no gaps) electrode, thanks to the gaps between the segmented boundaries. Hence, one expects a much larger total electroadhesion force for an interdigitated geometry than for a uniform geometry, as well as a force nearly uniformly distributed over the area for the interdigitated shape, but only a force localized at the periphery for the uniform shape. The gaps of the interdigitated electrode however lead to a lower electrostatic actuation due to the smaller overlapping electrode area across the DEA membrane, compared to the uniform electrode. The gaps can be narrowed to maximize DEA actuation, but the gap size must be sufficiently large to avoid electrical breakdown in the passive layer insulating the segmented electrodes (of order $100 \mu \mathrm{m}$ gaps are needed to withstand $5 \mathrm{kV}$ potential difference, essentially resulting from the breakdown field strength of silicone elastomer, typically 50 to $100 \mathrm{~V} / \mu \mathrm{m})$.

We assess the impact of electrode geometry on electroadhesion and electrostatic actuation through characterization of experimental devices to address the trade-off and an optimized design for the gripper. Additionally, understanding characteristics on electroadhesion is important since our DEA design, a bilayer offset interdigitated electrodes, is essentially different from other existing electroadhesion devices which have electrodes on single surface. 
We use two electrode geometries for characterization: an interdigitated shape and a square shape.

We first investigate the spatial distribution of the electroadhesion force. We raster a small force probe (10 mm diameter) over test samples with active electrode area of $40 \mathrm{~mm} \times 40 \mathrm{~mm}$ in a passive outline dimension of $70 \mathrm{~mm} \times 70 \mathrm{~mm}$ (Figure 3a,b), while recording the normal component of local electroadhesion force. The thickness of the top and the bottom passive layers are $50 \mu \mathrm{m}$. There is no measurable stiction between the passive layer and the probe interface. Figure $3 \mathrm{c}$ plots the measured local normal adhesion force at applied voltage of 2.5 $\mathrm{kV}$, and clearly represents the effect of the interdigitated design over the square design. The square electrode generates a local normal force of approximately $15 \mathrm{mN}$ only at the periphery, and a force of less than $0.1 \mathrm{mN}$ for all interior points. For the interdigitated electrode, the local force is much larger and homogeneous over the electrode surface; $\sim 120 \mathrm{mN}$ on average for all the points on the electrode, more than 1000 times larger force at the interior points compared to the square design. The result proves that the scaling of the electroadhesion force is proportional to the total electrode area for the interdigitated electrode, but only proportional to the periphery length for the square electrode. Also, for the gripper, the uniform electrode configuration will not enable holding an object smaller than the overall electrode area due to the absence of electroadhesion force at the center. The total electroadhesion force of the interdigitated design can be calculated by dividing the device electrode area $\left(1600 \mathrm{~mm}^{2}\right)$ by the probe area $\left(78.5 \mathrm{~mm}^{2}\right)$, and multiplying the measured local normal force $(\sim 120 \mathrm{mN})$, as this design scales the adhesion force proportional to area. Similarly, the total adhesion force of the square design can be obtained from the total periphery of the device $(160 \mathrm{~mm})$, the probe diameter $(10 \mathrm{~mm})$, and the measured maximum local normal force $(\sim 15 \mathrm{mN})$, based on the fact that this design generates the force only at the electrode periphery. The interdigitated 
electrode produces 10 times higher total electroadhesion force $(\sim 2400 \mathrm{mN})$ than to the square electrode $(\sim 240 \mathrm{mN})$.

According to the literature, the adhesion force scales with the square of the fringe electric field intensity that induces surface charges by polarization for dielectric materials ${ }^{[47,48]}$, and by electrostatic induction for conductive materials ${ }^{[48]}$. We investigate the influence of two important parameters - applied voltage $(0 \mathrm{~V}, 2.5 \mathrm{kV}$, and $5 \mathrm{kV})$ and bottom passive layer thickness $(50 \mu \mathrm{m}$ and $400 \mu \mathrm{m})$ - on the magnitude of the electroadhesion force, using simplified devices shown in Figure 4a that represent one finger of the gripper with active overall electrode area of $10 \mathrm{~mm} \times 10 \mathrm{~mm}$ with passive outline dimension of $15 \mathrm{~mm} \times 15 \mathrm{~mm}$. These devices are smaller than those previously discussed, but are the same size as the gripping part of the gripper, and allow using a probe covering the entire electrode area (10 $\mathrm{mm} \times 10 \mathrm{~mm}$ ) to measure the electroadhesion force in both shear force required to displace the probe, and normal force to detach it from the device. The measured shear force plotted in Figure $4 \mathrm{~b}$ scales with the voltage and the inverse of the thickness, in the manner shown in the literatures on conventional electroadhesion devices ${ }^{[32,49-52]}$. For the thinnest passive layer, the shear force at $5 \mathrm{kV}$ is $3.5 \pm 0.3 \mathrm{~N}$ (corresponds to $35 \pm 3 \mathrm{kPa}$ given the $10 \mathrm{~mm} \times 10 \mathrm{~mm}$ overall electrode area) for the interdigitated electrode, and $1.8 \pm 0.1 \mathrm{~N}(18 \pm 1 \mathrm{kPa})$ for the square electrode. The shear forces at zero applied voltage are due to static friction. The measured normal force (Figure 4c) scales with both the voltage and the inverse of the thickness in the same way as the shear force does. Since there is no inherent stiction between the passive layer and the interface, no normal force is seen at zero voltage. The normal force at $5 \mathrm{kV}$ is $1.3 \pm 0.2 \mathrm{~N}(13 \pm 2 \mathrm{kPa})$ for the interdigitated electrode, and $0.7 \pm 0.1 \mathrm{~N}(7 \pm 1 \mathrm{kPa})$ for the square electrode. The factor of two between measured electroadhesion forces for interdigitated and square electrodes is consistent with the ratio of edge lengths producing fringing fields for the two configurations. For a fixed electrode area, one could increase the 
electroadhesion force dramatically by patterning smaller gaps between the lines to enable longer total edge length. One is ultimately limited by fabrication resolution, electrical breakdown in small gaps, and the reduced penetration depth of the fringing in the object, since the fringing distance scales with electrode gap.

We then characterize the actuation stroke angle and the blocked force as functions of the applied voltage, using the same simplified devices with the bottom layer thickness of $400 \mu \mathrm{m}$ (Figure 4a). Figure 4d,e plot the measured actuation stroke angle and the blocked force, and show that the reduction of the actuation performance for the interdigitated electrode due to the gaps is only $\sim 20 \%$ compared to the square electrode. The reduction corresponds to smaller electrode overlap area; $83.75 \mathrm{~mm}^{2}$ in the interdigitated electrode, and $100 \mathrm{~mm}^{2}$ in the square electrode for the simplified devices. The magnitude of the blocked force is in the $\mathrm{mN}$ order, 3 orders of magnitude smaller than the electroadhesion force, representing the low mechanical grasping force of the gripper allowing handling of fragile and deformable objects, while the holding force is provided mostly by the shear electroadhesion force. The fact that 10 times higher electroadhesion force with only $20 \%$ reduction of the actuation performance can be generated from the interdigitated electrode design clearly shows the advantage for this design.

We fabricate the two-finger soft gripper (Figure 1) as a proof of concept to demonstrate its versatility at picking up and releasing different types of objects. The gripper structure, shown in Figure 1a, is realized as a planar extension of the DEA design (Figure 2a). This simple structure is lightweight $(\sim 1.5 \mathrm{~g})$ and allows for fast movement (it takes $\sim 100 \mathrm{~ms}$ to close the fingers, Supplementary Video S1). The geometry of the electrodes is designed to provide stronger adhesion forces around the fingertips by the fine interdigitated segmentation, and the other sections generate larger actuation stroke. The gripper is successful at handling various objects (Figure 1b-f). The electroadhesion force ensures adaptation of the actuated compliant 
gripper fingers, and realizes unprecedented functionality and performance in the device. The large holding force provided by the shear electroadhesion force (on the order of Newtons, Figure 4b) and the low mechanical grasping force provided by the actuation (on the order of milli-Newtons, Figure 4e) allow for easy handling of fragile objects such a raw chicken egg (Figure 1b, and Supplementary Video S1). The normal electroadhesion force allows for picking up flat objects such as a paper (Figure 1d, and Supplementary Video S1) without grasping, which is difficult for other finger-based grippers given the absence of features that can be grabbed. The functionality of the gripper is most impressively highlighted when the object is deformable (Figure 1c). The low mechanical grasping force minimizes the object's deformation, the normal adhesion force keeps the gripper's fingers in conformal contact with the target as it deforms, and the shear adhesion force provides the holding force. The deformable object, a water-filled silicone membrane balloon, is not only highly deformable but also very fragile (membrane thickness of $25 \mu \mathrm{m}$ ), and it breaks easily when picked up by the human hand. These challenging features make grasping extremely difficult, and handling of such highly sensitive objects with a gripper has not been reported. Picking up of slippery objects (Teflon tube, Figure 1e), can be difficult for other grasping devices and the Geckoinspired adhesion technology ${ }^{[42]}$. Handling of a metallic oil can (Figure 1f) proves that the electroadhesion is suited for conductive objects as well as dielectric objects.

We have described a method to create a lightweight, fast, all-silicone, soft gripper, based on a novel DEA design simultaneously maximizing electroadhesion and electrostatic actuation. The fact that handling of such a wide, unprecedented range of challenging objects is achieved by a single device with simple control input (Figure 1b-f), is a main advantage and novelty of our gripper over other existing grippers. The novel DEA design shows advantage for grasping application over traditional DEA design as it can produce up to 10 times higher electroadhesion forces at the cost of only $\sim 20 \%$ reduction in actuation performance. The total 
electroadhesion force generated from our DEA design, a bilayer offset interdigitated electrodes, can be much higher (order of several times) by optimizing the electrode geometry such as the gap and the segmented electrode width, and the thickness of the passive layer (Figure 4b-c), as has been suggested in electroadhesion devices with single surface electrode $^{[50,52-54]}$. The sensing capability of the device (Supplementary Figure S2) makes it an active smart skin enabling shape recognition to understand the object geometry when such information cannot be provided. The interdigitated electrodes can also be used to measure dielectric properties ${ }^{[55]}$ of the object and thus gain insight into its composition. The soft gripper technology described here has features that make it suitable for many applications. The lightweight feature encourages using the device in small transportation drones ${ }^{[56]}$, and the use of silicones i.e., a biocompatible material allows grasping tasks in food industry and medical (e.g., tissue handling ${ }^{[57]}$ ). The simplicity of the gripper structure has a high design flexibility able to adapt this method to produce a wide range of grippers of different size and shape, for example with 3 long fingers. The interdigitated electrode geometry helps the scaling by homogeneous distribution of adhesion forces (Figure 3c), and also allows locally tailoring the electrode geometry parts responsible for the adhesion and the actuation at different desired locations (e.g., our gripper shown in Figure 1a). As a multifunctional polymer actuator, the DEA technology described here could enable other soft robots beyond grippers. The actuation and the controllable high friction force (i.e., electroadhesion force) on the ground surface yield kinematically more efficient locomotion in bioinspired caterpillar robots $^{[58]}$ and gait robots ${ }^{[59]}$, and enable their wall-climbing functionality (an example of rigid robots in $\left.{ }^{[60]}\right)$. Soft modular robots ${ }^{[61]}$ are another promising application where the adhesion works as their interconnection, and the actuation moves the connected structure, or localizes each robot. Finally, all the applications mentioned above can benefit from the simple, highly integrated system thanks to the multifunctionality of the DEA design-electroadhesion, 
actuation with different output characteristics, and sensing — as highlighted in the presented soft gripper.

\section{Experimental Section}

Fabrication of the experimental devices and the gripper

We used the silicone Nusil CF19-2186 to fabricate the elastomer membranes. After mixing the two components of Nusil CF19 per manufacturer recommendations, the silicone was blade-casted on Polyethylene terephthalate (PET) film using a variable gap applicator (Zehntner ZUA2000) and a film applicator coater (Zehntner ZAA2300), then cured for 60 min at $80^{\circ} \mathrm{C}$, The thickness depends on the applicator gap. DEA membranes of two thicknesses were used: $80 \mu \mathrm{m}$ and $60 \mu \mathrm{m}$ for the experimental devices and for the gripper, respectively. The membranes were pre-stretched uniaxially with a ratio of 1.2 , and mounted in a Polymethyl methacrylate (PMMA) holding frame using a silicone adhesive foil (Adhesives Research ARclear 8932EE) for electrode patterning. The passive silicone layers (Dow Corning Sylgard 184) were also produced with the same blade casting process, and different curing temperatures were used: $80^{\circ} \mathrm{C}$ and $150^{\circ} \mathrm{C}$ for the simplified devices and the gripper, respectively. The thickness of the membranes and the passive layers was measured using a white light transmission interferometer. We used the same composition, patterning method, and curing condition of the compliant electrode described in $^{[27]}$. We used oxygen plasma surface activation to chemically bond the passive layers onto the DEA membrane with patterned electrodes. The electrical connections were realized by filling a silver epoxy inside punched holes made in the structure, and pieces of conductive tape were placed. After the wiring, the device was separated from the holding frame.

\section{Electroadhesion measurement:}

The experimental setup for shear and normal force measurements (Figure $4 b, c)$ is schematically shown in Supplementary Figure S3. The bending part of the simplified devices 
(free standing part) was flattened and fixed on to a PMMA plate, and on the top, a probe consisted of an interface material and PMMA is placed. We used a thin paper as the interface between the device and the probe to avoid any influence of stiction to the bottom passive layer in contact. The probe was connected to a motorized stage (Zaber T-LSR150B) via a load cell (ATI Industrial Automation Nano 17), string, and a spring. The shear force and the normal force were taken as the maximum detachment force when the interface was pulled away in horizontal and vertical direction, respectively. For the local normal force measurement (Figure 3), a probe of $10 \mathrm{~mm}$ diameter and a low capacity load cell (Applied Measurements UF1-55) were used. A high voltage supply (Auckland Biomimetics Lab EAP controller) was used to activate the devices.

Actuation stroke and blocked force measurements:

We used a CCD camera and image processing in MATLAB to determine the tip angle of the simplified gripper devices to characterize the actuation stroke angle (Figure $4 \mathrm{~d}$ inset). For the blocked force measurement, a load cell (Futek LRF400) was used (Figure 4e inset). A high voltage supply (Auckland Biomimetics Lab EAP controller) was used to activate the devices.

\section{Gripper demonstration:}

The picking up demonstration was performed using a motorized stage (Zaber T-LSR150B) controlled via a laptop computer, where the gripper was mounted using a silicone tube and PMMA parts. A high voltage supply (Auckland Biomimetics Lab EAP controller) was used to activate the device.

Fabrication of fragile, highly deformable water-filled thin membrane balloon:

A soft silicone membrane (Bluestar Silicones Silbione LSR 4305) of thickness $25 \mu \mathrm{m}$ was produced using the same fabrication process as for the DEA membranes, and mounted in a PMMA circular holding frame using a silicone adhesive foil (Adhesives Research ARclear 8932EE) without any pre-stretch. Water was poured on the membrane, resulting in a bowl 
shape due to weight of the water. The membrane was carefully twisted to close the opening, forming a balloon which was sealed using a silicone glue (Dow Corning 734 flowable sealant).

\section{Acknowledgements}

The authors thank O. A. Araromi, S. Mintchev, S. Chakraborty, J. E. Auerbach, and D. G. Courtney for their helpful comments and discussions. This work was supported by the Swiss National Centre of Competence in Research (NCCR) Robotics, and the Swiss National Science Foundation grant 200020-153122.

[1] A. Bicchi, IEEE Trans. Robot. Autom. 2000, 16, 6.

[2] G. J. Monkman, S. Hesse, R. Steinmann, H. Schunk, Robot Grippers, Weinheim: WileyVCH Verlag GmbH and Co. KGaA, Germany 2006.

[3] G. Carbone, Grasping in Robotics, Springer-Verlag, London, UK 2013.

[4] R. Pfeifer and J. Bongard, How the Body Shapes the Way We Think: A New View of Intelligence, MIT Press, Cambridge, MA, USA 2006.

[5] S. Hirai, T. Tsuboi, T. Wada, in IEEE International Symposium on Assembly and Task Planning, Fukuoka, Japan 2001.

[6] A. M. Howard, G. A. Bekey, Auton. Robots 2000, 9, 1.

[7] A.-M. Cretu, P. Payeur, E. M. Petriu IEEE Trans. Syst. Man, Cybern. B, Cybern. 2012, 42, 3.

[8] H. Lin, F. Guo, F. Wang, Y.-B. Jia, Int. J. Robot. Res. DOI: 10.1177/0278364914564232.

[9] D. Henrich, H. Wörn, Eds., Robot Manipulation of Deformable Objects, Springer-Verlag, London, UK 2000.

[10] G. Fantoni, M. Santochi, G. Dini, K. Tracht, B. Scholz-Reiter, J. Fleischer, T. K. Lien, G. Seliger, G. Reinhart, J. Franke, H. N. Hansen, A. Verl, CIRP Ann. Manuf. Techn. 2014, 63, 2. 
[11] S. Bauer, S. Bauer-Gogonea, I. Graz, M. Kaltenbrunner, C. Keplinger, R. Schwödiauer, Adv. Mater. 2014, 26, 1.

[12] D. Rus, M. T. Tolley, Nature 2015, 521, 7553.

[13] M. L. Hammock, A. Chortos, B. C.-K. Tee, J. B.-H. Tok, Z. Bao, Adv. Mater. 2013, 25, 42.

[14] T. Rienmüller, H. Weissmantel, in 18th International Symposium on Industrial Robots, Lausanne, Switzerland 1988.

[15] E. Brown, N. Rodenberg, J. Amend, A. Mozeika, E. Steltz, M. R. Zakin, H. Lipson, H. M. Jaeger, Proc. Natl. Acad. Sci. U.S.A. 2010, 107, 44.

[16] G. L. Kenaley, M. R. Cutkosky, in IEEE International Conference on Robotics and Automation, Scottsdale, AZ, USA 1989.

[17] G. J. Monkman, Robotica 1992, 10, 2.

[18] G. J. Monkman, P. M. Taylor, in 5th International Conference on Advanced Robotics, Pisa, Italy 1991.

[19] F. Ilievski, A. D. Mazzeo, R. F. Shepherd, X. Chen, G. M. Whitesides, Angew. Chem. Int. Ed. Engl. 2011, 50, 8.

[20] D. Petković, N. D. Pavlović, S. Shamshirband, N. B. Anuar, Ind. Robot 2013, 40, 6, 2013.

[21] S. Song, M. Sitti, Adv. Mater. 2014, 26, 28.

[22] R. Deimel, O. Brock, in Robotics:Science and Systems, Berkeley, CA, USA, 2014.

[23] O. A. Araromi, I. Gavrilovich, J. Shintake, S. Rosset, M. Richard, V. Gass, H. Shea, IEEE/ASME Trans. Mechatronics 2015, 20, 1.

[24] S. Shian, K. Bertoldi, D. R. Clarke, Adv. Mater. (Forthcoming).

[25] R. Pelrine, R. Kornbluh, Q. Pei, J. Joseph, Science, 2000, 287, 5454.

[26] I. A. Anderson, T. A. Gisby, T. G. McKay, B. M. O’Brien, E. P. Calius, J. Appl. Phys. 2012, 112, 4 .

[27] L. Maffli, S. Rosset, M. Ghilardi, F. Carpi, H. Shea, Adv. Funct. Mater. 2015, 25, 11. 
[28] S. Rosset and H. Shea, in SPIE Electroactive Polymer Actuators and Devices (EAPAD), San Diego, CA, USA 2015.

[29] T. A. Gisby, B. M. O’Brien, I. A. Anderson, Appl. Phys. Lett. 2013, 102, 19.

[30] S. Rosset, H. Shea, Appl. Phys. A Mater. Sci. 2012, 110, 2.

[31] R. E. Pelrine, R. D. Kornbluh, J. P. Joseph, Sens. Actuator A-Phys. 1998, 64, 1.

[32] K. Asano, F. Hatakeyama, K. Yatsuzuka, IEEE Trans. Ind. Appl. 2002, 38, 3.

[33] H. Wang, A. Yamamoto, T. Higuchi, Int. J. Adv. Robot. Syst. 2014, 11, 191.

[34] H. Prahlad, R. Pelrine, S. Stanford, J. Marlow, R. Kornbluh, in IEEE International

Conference on Robotics and Automation, Pasadena, CA, USA 2008.

[35] G. Monkman, Ind. Robot 2003, 30, 4.

[36] Grabit Inc., "Products", http://grabitinc.wpengine.com/products/\#panelgrippers, accessed; May, 2015.

[37] M. P. Murphy, B. Aksak, M. Sitti, Small 2009, 5, 2.

[38] S. Kim, M. Spenko, S. Trujillo, B. Heyneman, D. Santos, M. R. Cutkosky, IEEE Trans. Robot. 2008, 24, 1.

[39] A. Parness, D. Soto, N. Esparza, N. Gravish, M. Wilkinson, K. Autumn, M. Cutkosky, J. R. Soc. Interface 2009, 6, 41.

[40] S. A. Suresh, D. L. Christensen, E. W. Hawkes, M. Cutkosky, J. Mech. Robot. 2015, 7, 2.

[41] E. W. Hawkes, D. L. Christensen, A. K. Han, H. Jiang, M. R. Cutkosky, in IEEE International Conference on Robotics and Automation, Seattle, WA, USA 2015.

[42] K. Autumn, A. M. Peattie, Integr. Comp. Biol. 2002, 42, 6.

[43] G. Monkman, P. Taylor, G. Farnworth, Int. J. Cloth Sci. Tech. 1989, 1, 3.

[44] I. D. Johnston, D. K. McCluskey, C. K. L. Tan, M. C. Tracey, J. Micromech. Microeng. $\mathbf{2 0 1 4}, 24,3$.

[45] J. Shintake, S. Rosset, B. Schubert, S. Mintchev, D. Floreano, H. Shea, in SPIE Electroactive Polymer Actuators and Devices (EAPAD), San Diego, USA 2015. 
[46] G. Kofod, W. Wirges, M. Paajanen, S. Bauer, Appl. Phys. Lett. 2007, 90, 8.

[47] H. A. Pohl, J. Appl. Phys.1958, 29, 8.

[48] A. D. Moore, Electrostatics and its applications, Wiley, NY, USA 1973.

[49] A. Yamamoto, T. Nakashima, T. Higuchi, in IEEE International Symposium on MicroNanoMechatronics and Human Science, Nagoya, Japan 2007.

[50] R. Liu, R. Chen, H. Shen, R. Zhang, Int. J. Adv. Robot. Syst. 2013, 10, 36.

[51] V. Sabermand, Y. Hojjat, M. Hasanzadeh, Int. J. Electrical, Comp. Electron. Common, Engineer. 2014, 8, 11.

[52] K. Yatsuzuka, F. Hatakeyama, K. Asano, S. Aonuma, IEEE Trans. Ind. Appl. 2000, 36, 2.

[53] I. Donald Ruffatto, J. Shah, M. Spenko, in ASME 2012 International Design Engineering Technical Conferences and Computers and Information in Engineering Conference, Chicago, IL, USA 2012.

[54] D. Ruffatto III, J. Shah, M. Spenko, J. Electrostat. 2014, 72, 2.

[55] A. V. Mamishev, K. Sundara-Rajan, F. Yang, Y. Du, M. Zhan, Proc. IEEE 2004, 92, 5.

[56] D. Floreano, R. J. Wood, Nature 2015, 521, 7553.

[57] A. Vankov, P. Huie, M. Blumenkranz, D. Palanker, in SPIE Ophthalmic technologies, Bellingham, WA, USA 2004.

[58] H.-T. Lin, G. G. Leisk, B. Trimmer, Bioinspir. Biomim. 2011, 6, 2.

[59] R. F. Shepherd, F. Ilievski, W. Choi, S. A. Morin, A. A. Stokes, A. D. Mazzeo, X. Chen, M. Wang, G. M. Whitesides, Proc. Natl. Acad. Sci. U.S.A. 2011, 108, 51.

[60] R. Chen, R. Liu, J. Chen, J. Zhang, in IEEE International Conference on Robotics and Biomimetics, Shenzhen, China 2013.

[61] J. Germann, M. Dommer, R. Pericet-Camara, D. Floreano, Adv. Robot. 2012, $26,7$. 
a

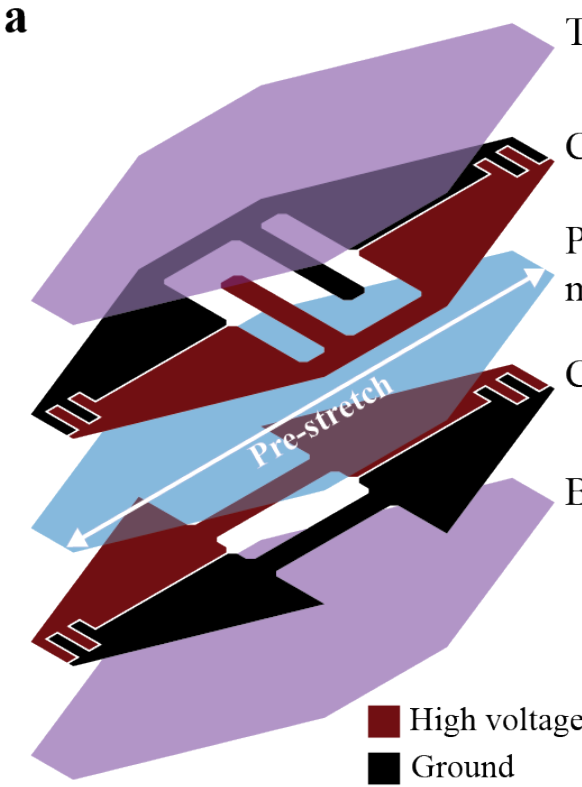

Top passive layer $(100 \mu \mathrm{m})$

Compliant electrode

Pre-stretched elastomer

membrane $(60 \mu \mathrm{m})$

Compliant electrode

Bottom passive layer $(175 \mu \mathrm{m})$

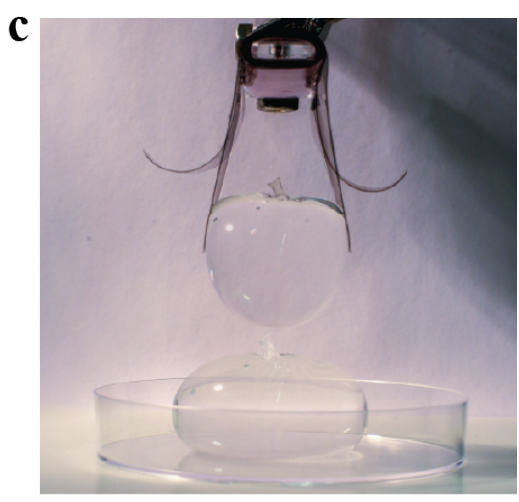

d
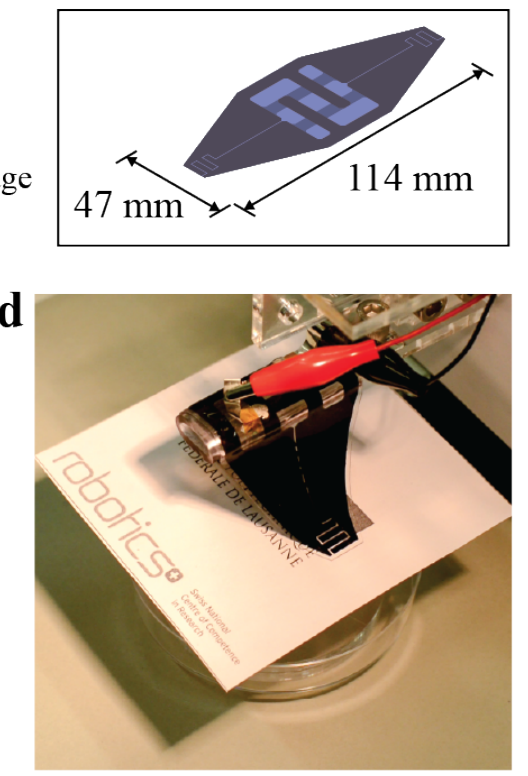

e
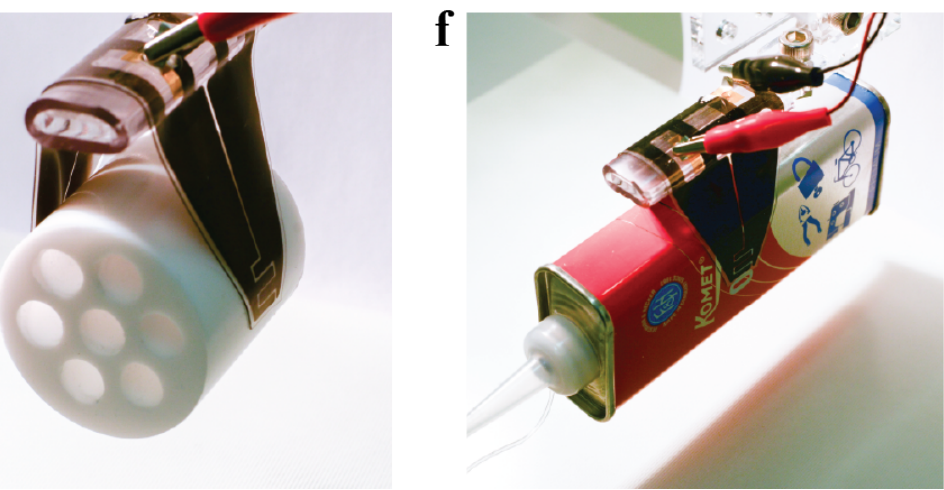
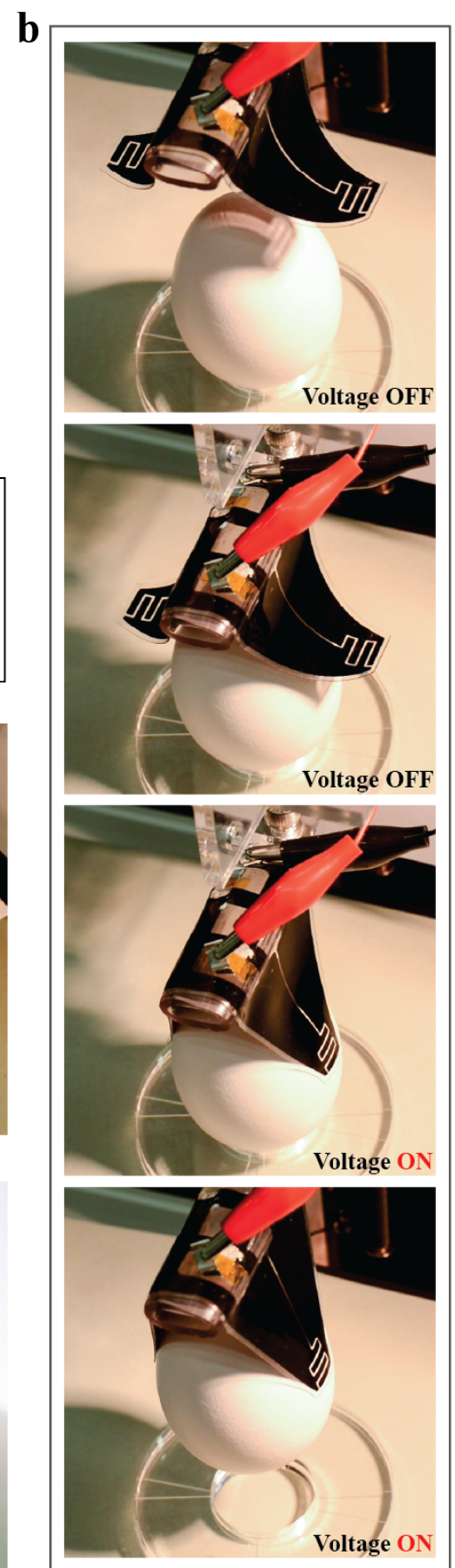

Figure 1. Structure of the electroadhesion-enabled soft gripper, and demonstration of gripping different objects. (a) The gripper consists of a pre-stretched elastomer membrane with patterned compliant electrodes laminated between two passive silicone layers. Different colors of the electrodes represent their polarity (red is positive, and black is negative). (b)

Picking up objects is performed using an external motorized stage where the gripper is raised and lowered by an external motorized stage to act as an end effector (see also Supplementary 
Video S1). The gripper has open fingers (curled shape) at zero applied voltage. When the voltage is applied $(3.5 \mathrm{kV})$, the fingers are actuated to close around the object, here a raw chicken egg (60.9 g), while the electroadhesion force is simultaneously generated. The object is then picked up thanks to the holding force provided by the electroadhesion while the motorized stage is moved. Releasing of the object is achieved by a reverse of the operating procedure explained above. With the same operating principle, picking up demonstration is performed for (c) a fragile, highly deformable water-filled thin membrane balloon (35.6 g), (d) a flat paper (0.8 g), (e) a Teflon tube (80.8 g), and (f) a metallic oil can (82.1 g).

Conventional grippers cannot manipulate such a wide range of challenging objects.

a

$$
\text { Top passive layer }
$$

Elastomer membrane

Bottom passive layer

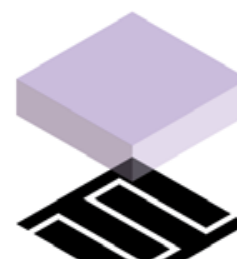

(1)

r

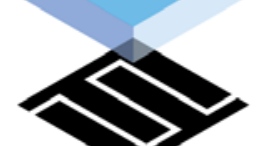

er

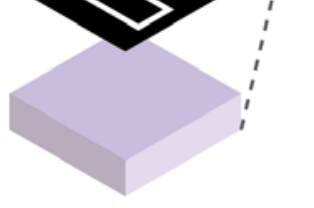

d

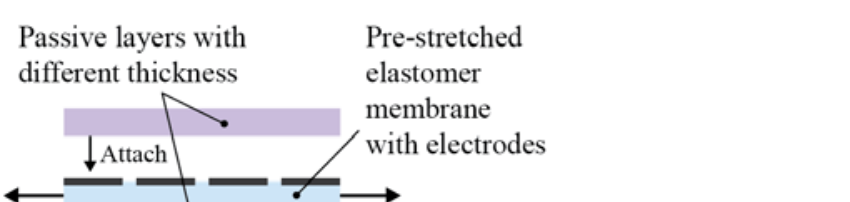

Cross-section

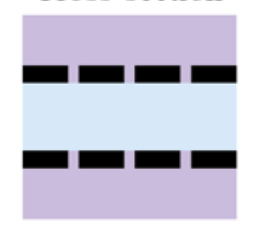

b
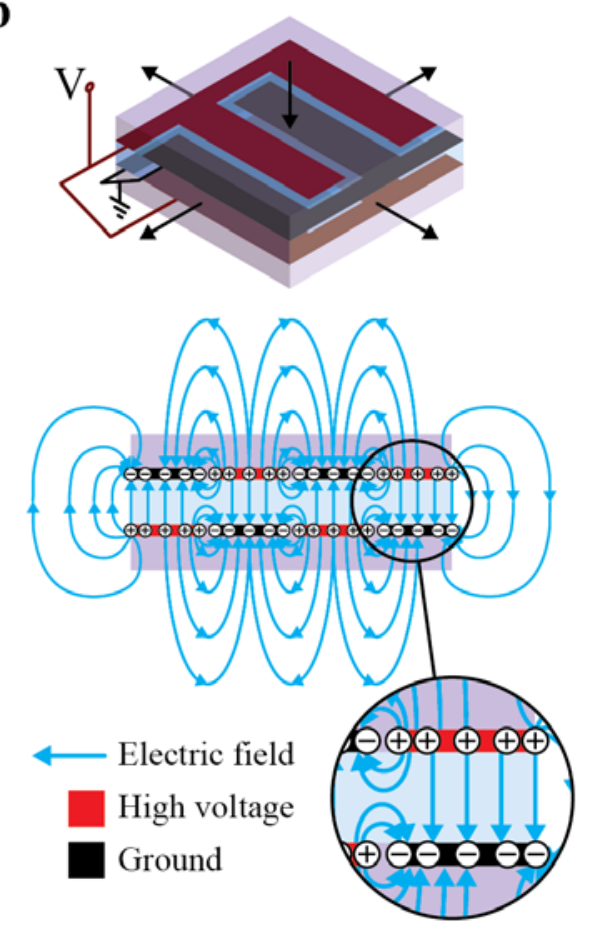

Electric field

High voltage

Ground

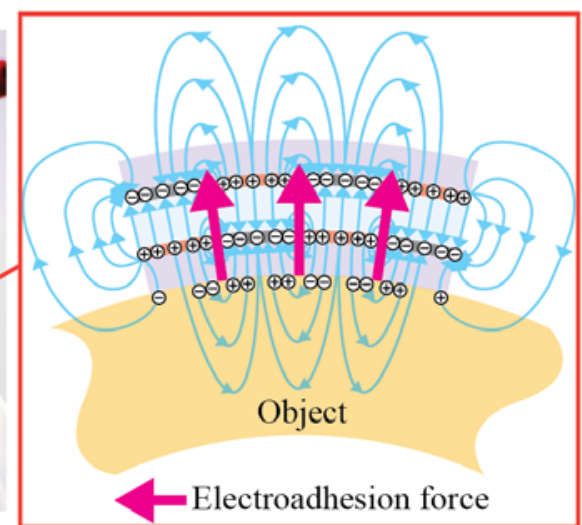


Figure 2. Operating principle of the novel DEA design for the gripper. (a) Each of the electrodes sandwiching the elastomer membrane consists of two interdigitated electrodes. Two passive layers made of a silicone are laminated on the top and bottom. The bottom passive layer touches the object being picked up. (b) DEA actuation (bending motion in this multilayer configuration) is proportional to the square of the electric field in the membrane in the direction normal to the surface, and the electroadhesion force is proportional to the square of the fringe electric fields. For optimum combined DEA and electroadhesion operation, one applies the same voltage difference between the neighboring interdigitated electrodes on the top and bottom layers, but laterally offset so as to obtain an alternating high electric field normal to the membrane (where top and bottom electrodes overlap), and strong fringe fields in the gaps at the segmentation boundaries. Other electrodes configurations lead to different actuation and gripping modes (Supplementary Figure S1). (c) These fringe fields create electroadhesion forces on the object surface in contact, which are used to generate substantial holding forces in the gripper. The electroadhesion is maximum in the region where the electrodes are interdigitated, allowing tailoring which parts of the electroadhesion grip, and parts which only bend. (d) DEA gripper consists of a pre-stretched DEA bonded to passive layers. The structure is curled at zero applied voltage. Applying voltage to the DEA generates electrostatic pressure, which reduces the stress in the DEA, uncurling the structure towards a flat shape. 
$\mathbf{a}$

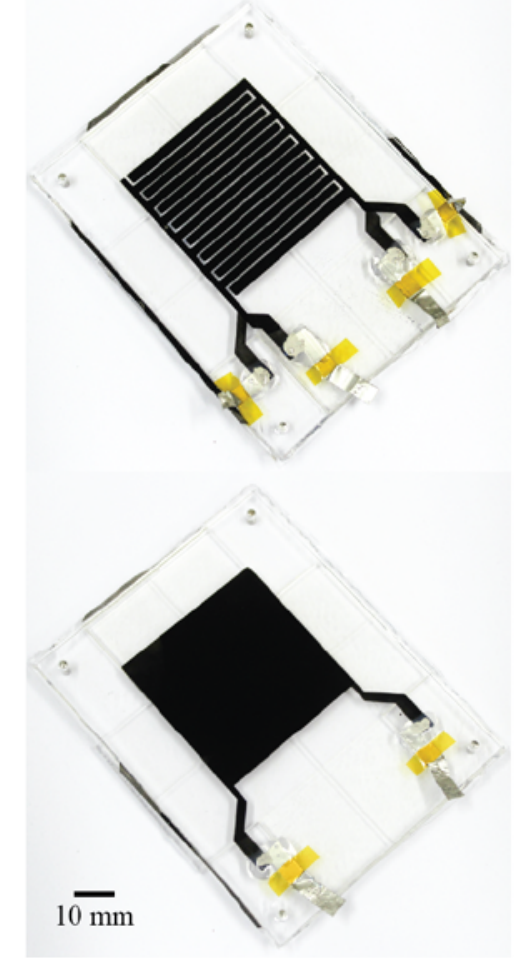

b
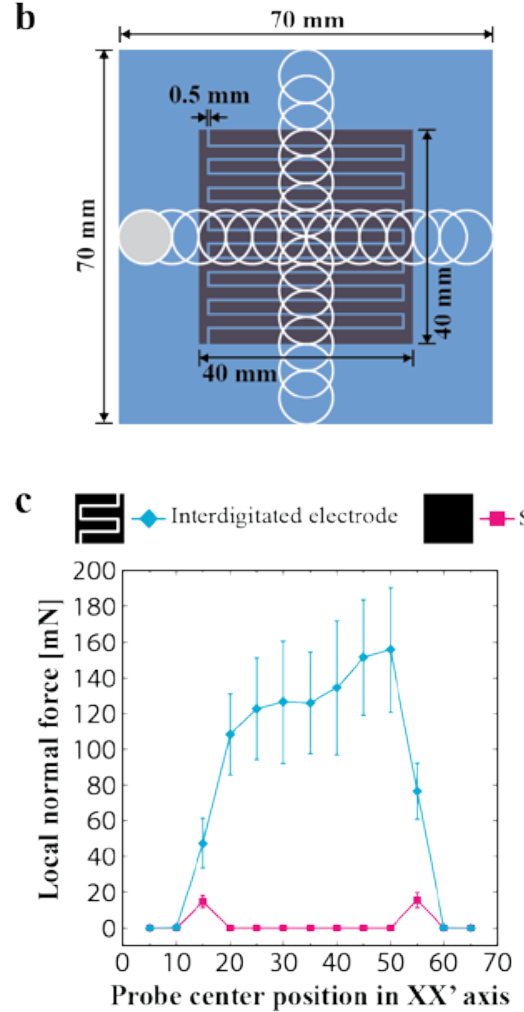

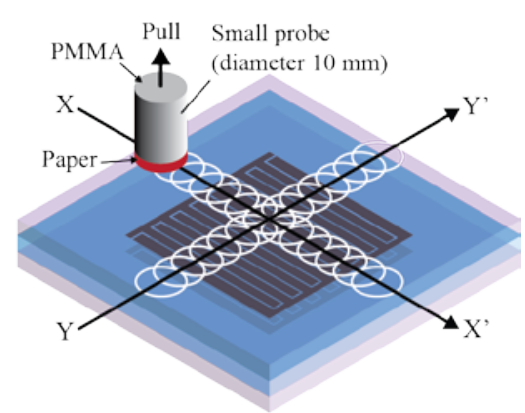

Square electrode

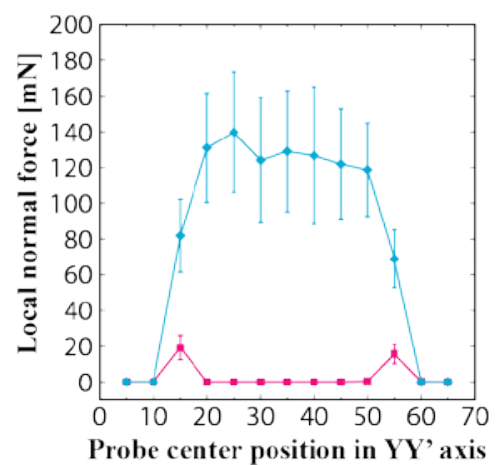

Figure 3. Characterization of the spatial dependence of the electroadhesion force. (a) The experimental devices used for the characterization (top: interdigitated, bottom: square).

Electrode area is $40 \mathrm{~mm} \times 40 \mathrm{~mm}$. (b) The local electroadhesion force is measured at 13 points along the lines crossing the center of the devices ( $\mathrm{XX}$ ' and $\mathrm{YY}^{\prime}$ axes) using a probe of diameter $10 \mathrm{~mm}$. (c) (left) The measured local normal electroadhesion force as a function of the probe position for an applied voltage of $2.5 \mathrm{kV}$ in $\mathrm{XX}^{\prime}$ axis, and (right) in $\mathrm{YY}^{\prime}$ axis. The interdigitated electrode generates much larger and more homogeneous local forces over the electrode surface thanks to the segmented electrode boundaries, while the square electrode produces forces only at the periphery. For every measurement, 2 devices are characterized 3 times each, and the average force is reported. 
a
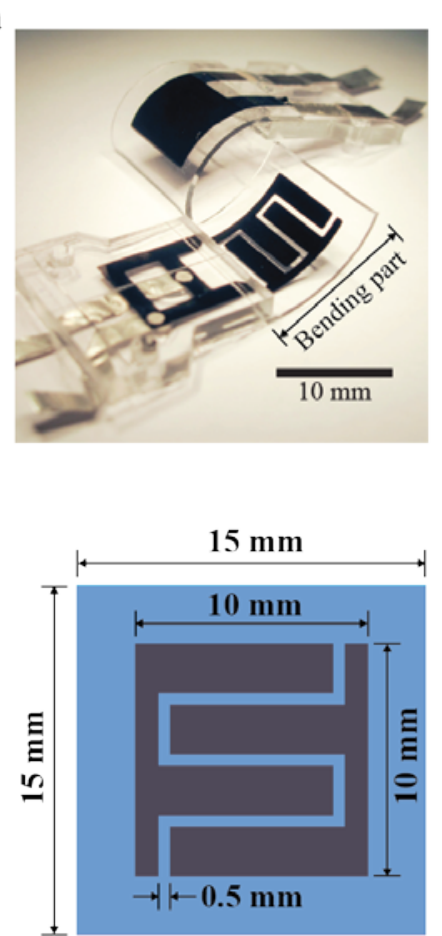

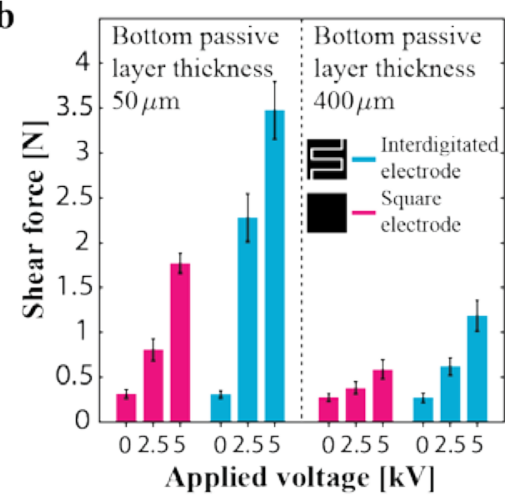

b

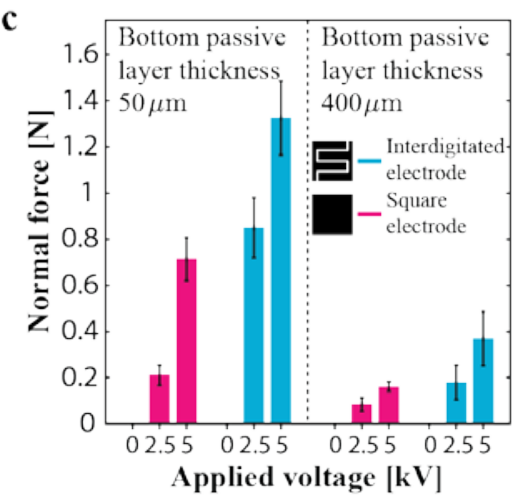

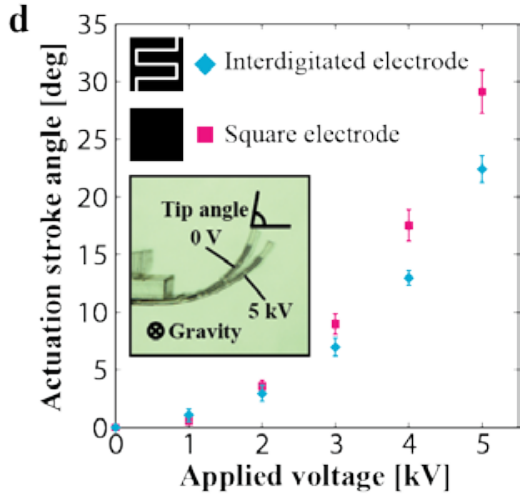

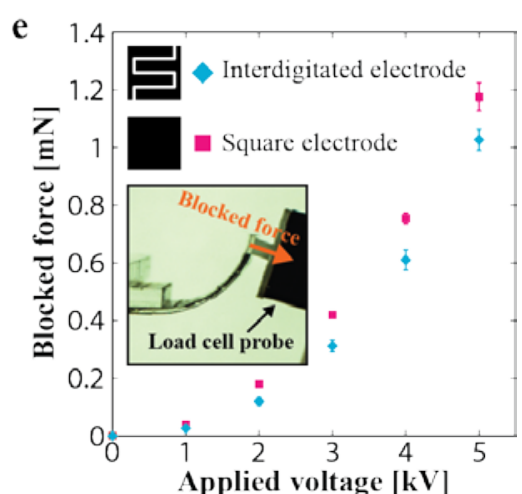

Figure 4. Characterization of the total electroadhesion force and of the actuation performance on simplified devices. (a) (top) The simplified devices used for the characterization (with 400 $\mu \mathrm{m}$-thick bottom passive layer facing to the interface), and (bottom) their dimension (bending part). The cross-section of the devices is shown in Supplementary Figure S3. (b) The measured electroadhesion shear force as a function of applied voltage for different bottom passive layer thicknesses. The shear force scales with both voltage and thickness: increasing the voltage and decreasing the thickness increase the adhesion force. The forces at $0 \mathrm{~V}$ are due to static friction. (c) The measured electroadhesion normal force as a function of the applied voltage for different passive layer thicknesses. The normal force scales with the voltage and the thickness in the same way as the shear force does. Since there is no inherent stiction between the passive layer and the interface, no normal force is seen at $0 \mathrm{~V}$. (d) The measured actuation stroke angle as a function of the applied voltage. The interdigitated electrode shows $\sim 20 \%$ lower actuation stroke compared to the square electrode. The inset shows the bending actuation of the device with the interdigitated electrodes. The actuation stroke is obtained as the tip angle difference from the initial angle. (e) The measured blocked force as a function of 
the applied voltage. A trend similar to the actuation stroke, i.e., $\sim 20 \%$ reduction in the interdigitated electrode is observed. The inset shows a sample being measured. The blocked force is obtained by putting the probe of a load cell on the tip of the samples at the initial shape. For every measurement, 4 devices are characterized 3 times each, and their average is taken. 


\section{Supplementary Figures}

a

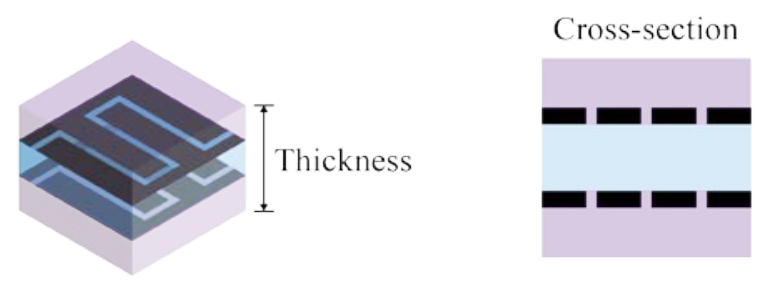

b
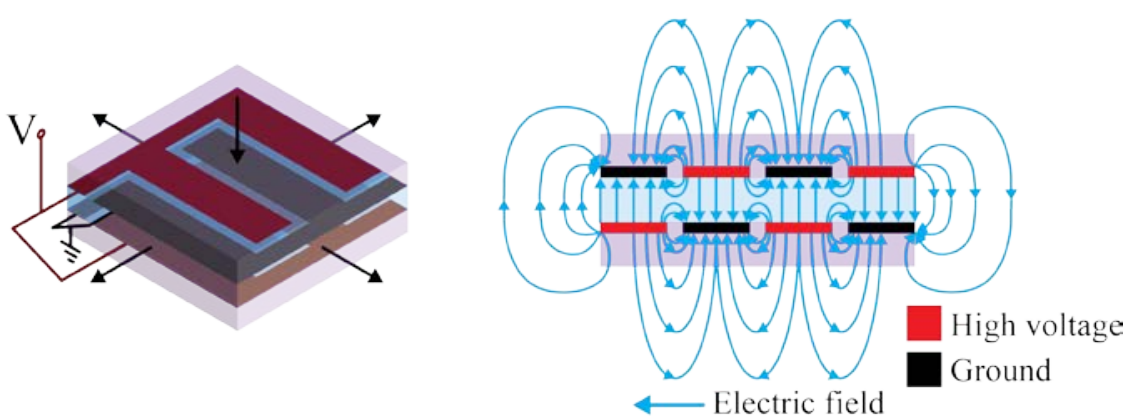

$\mathrm{C}$
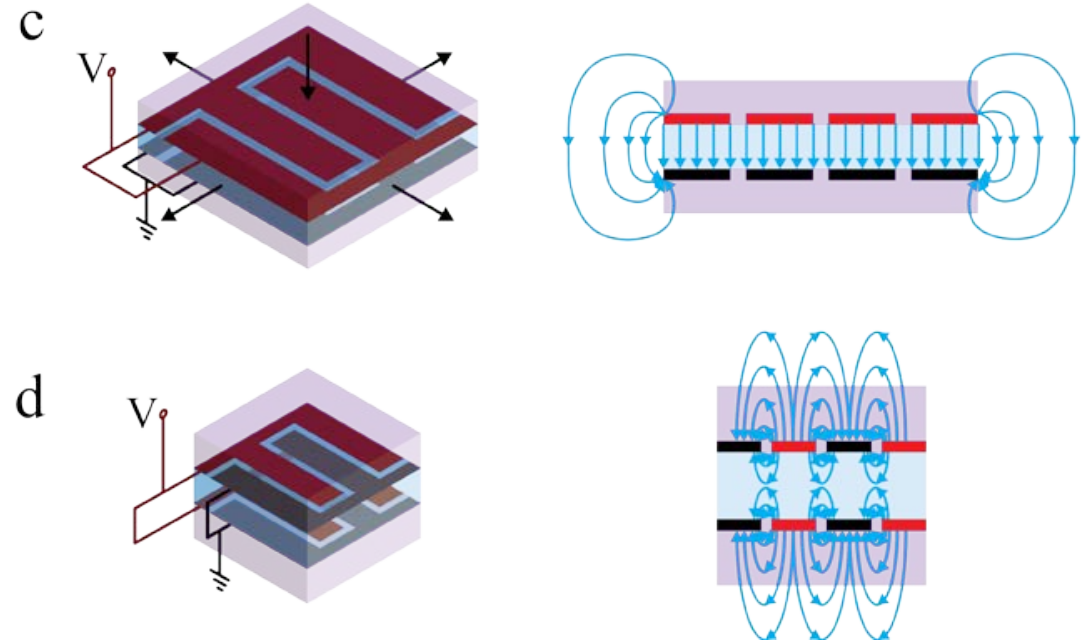

Figure S1. Different output characteristics of the novel DEA design. (a) Initial state of the DEA (without any applied voltage). (b) Activation with the wiring used for the gripper configuration (described in the main text), which creates electrostatic actuation with higher electroadhesion force. (c) Activation with opposite potentials across the thickness direction like usual DEAs results in electrostatic actuation with fringe electric fields at the edges, generating actuation with reduced electroadhesion force. (d) Activation with opposite potentials on the same planar surface and same potentials in the thickness direction results in fringe electric fields on the surface, i.e., electroadhesion forces while no electrostatic actuation is produced. 


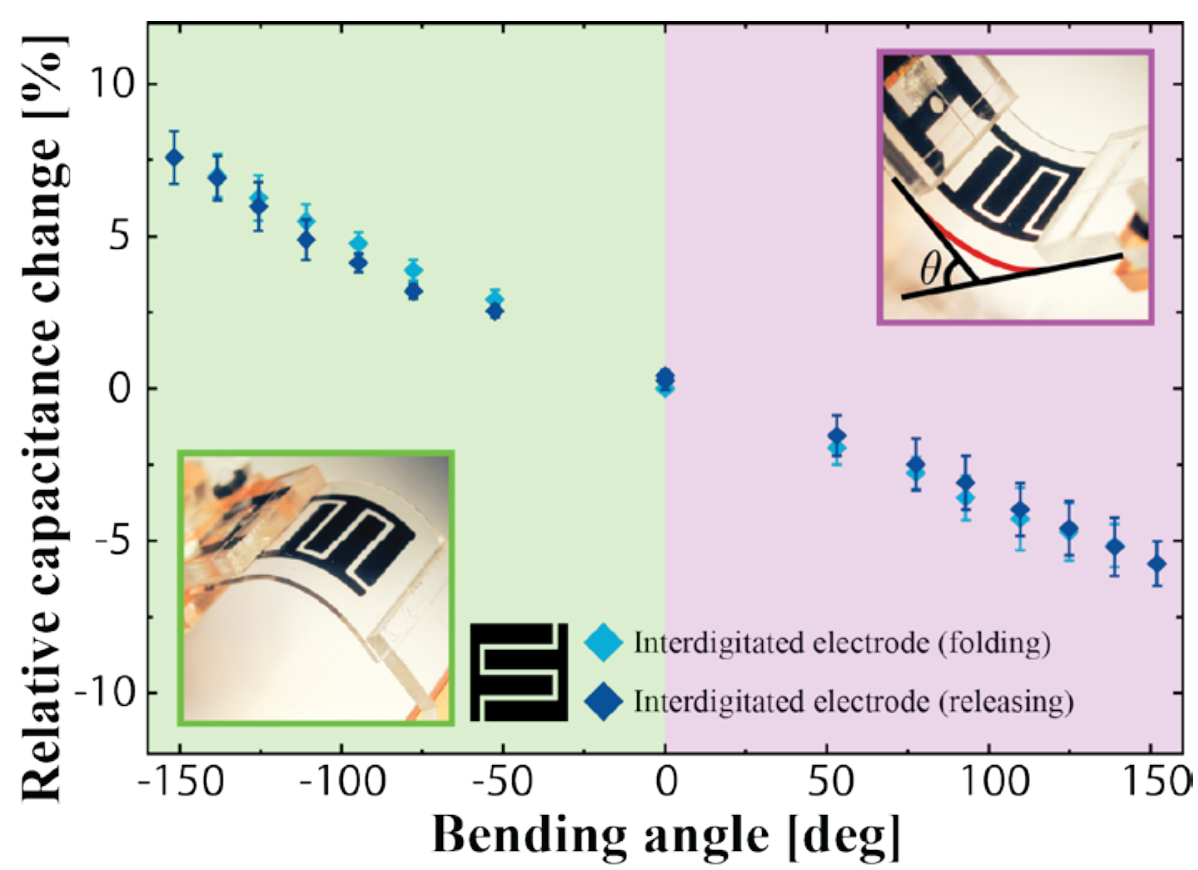

Figure S2. Measured capacitance as a function of the bending angle. The capacitance between the electrodes changes in response to bending of the structure (i.e., curvature).We measure the capacitance of the samples used in the characterization described in the main text. The samples are wired in the same way as the gripper configuration (Supplementary Figure S1b), where a LCR meter (Agilent E4980A) is used to measure capacitive changes. The structure is passively bent in two directions using a linear stage while the angle $\theta$ is measured with a CCD camera. The outline colors of the insets correspond to the positive or the negative bending angle which are shown in the corresponding color domains. Due to different thicknesses in the passive layers $(50 \mu \mathrm{m}$ and $400 \mu \mathrm{m})$, the devices show capacitance change in two bending directions up to $\pm 150^{\circ}$. In this measurement, 4 samples are measured 3 times each and their average is taken. The average capacitance at $0^{\circ}$ is $39.9 \mathrm{pF}$. 

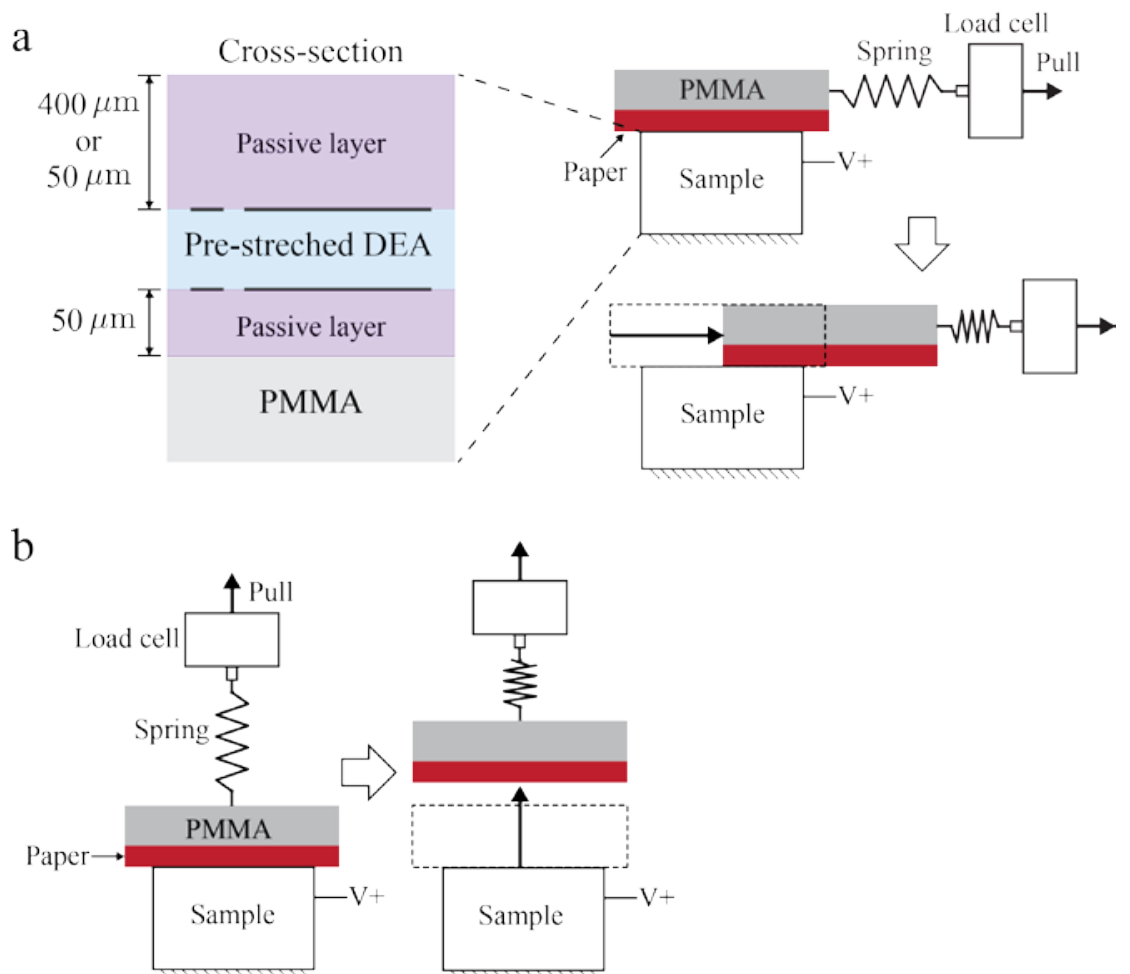

Figure S3. Electroadhesion force measurement. (a) The shear force is obtained as maximum detachment force when the interface part consisted of a paper and a PMMA plate is moved by an external motorized stage which is connected to the interface via a load cell, a spring, and a string. The sample is fixed on a mount. (b) With the same setup, the normal force is obtained by changing the moving direction of the interface part. 


\section{Supplementary Video}

Supplementary Video S1: Demonstration of the gripper picking up a broad range of objects (a raw chicken egg, a flat paper, and a water balloon). The gripper is raised and lowered by an external motorized stage to acts as an end effector.

https://goo.g1/IYXnX0 